\title{
RELIEF OF PAIN AND PARAESTHESIAE BY NERVE BLOCK DISTAL TO A LESION
}

\author{
BY \\ R. F. KIBLER* and P. W. NATHAN

\begin{abstract}
From the Neurological Research Unit of the Medical Research Council, the National Hospital, Queen Square, London, W.C.1
\end{abstract}

Lesions of afferent pathways may give rise to a variety of spontaneous sensations. When a lesion affects nerves, nerve roots, the root entry zone, or the spino-thalamic tract, the sensations are usually those of pain and painful pins and needles; when it affects the posterior columns of the cord, the sensation is one of painless pins and needles or of tingling. It is natural to think that the pain and paraesthesiae are due to the discharge of nerve fibres in or near the lesion, and that the lesion on the afferent pathway causes these nerve fibres to fire off.

In this paper, evidence will be presented to show that local anaesthetic injections of the afferent pathway, distal to the site of the lesion, may stop the pain or paraesthesiae; further, that this effect may far outlast the duration of the anaesthesia; and further, that blocking a peripheral nerve supplying a large part but not the whole of the region where the pain or paraesthesiae are felt may remove these sensations from the entire region.

A summary of the cases in which a nerve block distal to the lesion gave these results is presented as Table I. The cases are now briefly described and the experimental protocols are given.

\section{Results}

N.T. (National Hospital No. 80434.)-This 42-yearold woman was admitted to the National Hospital in November, 1958. In January, 1955, she had first noticed a cramping feeling and pins and needles in the right hand, extending up the outer aspect of the forearm and arm. Within one month of the onset of these paraesthesiae, moderate weakness of the limb developed. She was treated by traction of the neck and ordered to wear a sling. The strength of the limb returned to normal within a few months, but the paraesthesiae, although less intense, persisted till the time of admission. Six months before admission she found that her right leg and foot were becoming clumsy.

When she arrived in hospital, she described these paraesthesiae in the right upper limb as "irritating and

* Markle Scholar from the University of Pittsburgh. bothersome" but not as a true pain; she said that the skin in the affected region was unduly sensitive, anything rubbing it causing an increase in the paraesthesiae and making the sensation very unpleasant. She stated that hot and cold felt more intense in this area of skin, although she had burned the little finger without noticing any pain. The appreciation of light touch and two-point discrimination was diminished over all the fingers of the right hand and on the thumb; these changes were most marked in the thumb and index finger. The paraesthesiae of which she was complaining were aggravated by stroking or scratching the thumb, the index, the radial side of the forearm, the outer surface of the upper arm, and also a small area on the anterior chest well just below the clavicle. The right biceps jerk was diminished. All rapid movements of the right foot were impaired; and the plantar response on the right was the Babinski type.

The cerebrospinal fluid contained 6 lymphocytes and $0.8 \mathrm{mg}$. of protein per ml. Radiographs of the cervical spine, a myelogram, and an air encephalogram were normal

This patient's main symptoms and signs indicated involvement of several cervical roots or spinal segments of the cord on the right, and, in particular, the sixth cervical. The damage to the long tracts affecting the movements of the right lower limb had occurred more recently. It was thought most likely that the patient had disseminated sclerosis; damage to the nerve roots and spinal cord on the right side by prolapsed discs was considered as an alternative diagnosis.

Protocol of Experiment.-Before the injection of the right median nerve, the pins and needles sensation was confirmed to be present mainly in the palmar surface of the right thumb; the cramping sensation was present in the entire right hand. The sensations were described as unpleasant; they were aggravated by stroking.

The first attempt at injecting the median nerve above the elbow failed, the medial cutaneous of the forearm being injected in error. In one sense, this injection thus acted as a control. The patient knew she had had an injection, and she experienced some numbness in the limb but this injection had no effect on the paraesthesiae.

The median nerve was then injected with $1 \%$ lignocaine successfully just distal to the elbow. There ensued a rise in temperature and much diminution in sensibility: 
TABLE I

PATIENTS INVESTIGATED

\begin{tabular}{|c|c|c|c|c|c|c|}
\hline $\begin{array}{l}\text { Patient's } \\
\text { Initials }\end{array}$ & Diagnosis & $\begin{array}{l}\text { Site of Lesion } \\
\text { (presumed) }\end{array}$ & $\begin{array}{l}\text { Main Segments } \\
\text { where Pain or } \\
\text { Paraesthesiae } \\
\text { are Felt }\end{array}$ & $\begin{array}{c}\text { Kind of } \\
\text { Pain or } \\
\text { Paraesthesiae }\end{array}$ & $\begin{array}{c}\text { Structure } \\
\text { Blocked } \\
\text { by Local } \\
\text { Anaesthetic }\end{array}$ & Results \\
\hline N.T. & $\begin{array}{l}\text { Probably disseminated } \\
\text { sclerosis, possibly } \\
\text { prolapsed cervical } \\
\text { discs }\end{array}$ & Spinal roots & 6th cervical & $\begin{array}{l}\text { Unpleasant } \\
\text { pins and } \\
\text { needles and } \\
\text { sensation of } \\
\text { cramp }\end{array}$ & Median nerve & $\begin{array}{l}\text { Removal of pins and } \\
\text { needles and sensation of } \\
\text { cramp. They remained } \\
\text { absent several hours } \\
\text { after anaesthesia had } \\
\text { worn off. }\end{array}$ \\
\hline J.N. & $\begin{array}{l}\text { Carcinoma of } \\
\text { bronchus spreading } \\
\text { intc spinal canal }\end{array}$ & Spinal roots & $\begin{array}{l}\text { 7th and } 8 \text { th } \\
\text { cervical }\end{array}$ & Pain & Ulnar nerve & $\begin{array}{l}\text { Removal of pain. It re- } \\
\text { mained absent several } \\
\text { hours after anaesthesia }\end{array}$ \\
\hline G.N. & Disseminated sclerosis & Posterior root & 8th cervical & Burning pain & & Permanent relief of pain \\
\hline & Disseminated sclerosis & & 7 th and 8th & & Ulnar nerve & $\begin{array}{l}\text { Removal of pins and } \\
\text { needles }\end{array}$ \\
\hline H.G. & Syringomyelia & Posterior horn & 6 th, 7 th, and & Aching pain & Ulnar nerve & Removal of pain \\
\hline G.B. & $\begin{array}{l}\text { Intramedullary spinal } \\
\text { cord lesion }\end{array}$ & $\begin{array}{l}\text { Spinothalamic } \\
\text { tract }\end{array}$ & $\begin{array}{l}\text { 5th lumbar to } \\
\text { 5th sacral }\end{array}$ & Burning pain & Sacral roots & Removal of pain \\
\hline L.R. & $\begin{array}{l}\text { Carcinoma of cervix } \\
\text { uteri }\end{array}$ & Sacral roots & 5th lumbar to & Pain & Sciatic nerve & Removal of pain \\
\hline J.P. & $\begin{array}{l}\text { Prolapsed lumbar } \\
\text { discs }\end{array}$ & Spinal roots & $\begin{array}{l}\text { Inclusive } \\
\text { 3rd lumbar }\end{array}$ & Burning pain & $\begin{array}{l}\text { (a) Skin } \\
\text { (b) Femoral } \\
\text { nerve }\end{array}$ & $\begin{array}{l}\text { Removal of pain } \\
\text { Removal of pain; it re- } \\
\text { mained absent } 46 \text { hours } \\
\text { after anaesthesia had } \\
\text { worn off }\end{array}$ \\
\hline
\end{tabular}

the sharp and painful element of pin prick throughout the median distribution had gone, but touch and pressure were still felt. Both pins and needles and the cramping sensation disappeared completely. When the skin on the outer side of the right arm and on the anterior aspect of the chest was rubbed with the examiner's finger, the region which had previously given rise to the unpleasant dysaesthesiae, now felt "smooth and velvety"; it had no unpleasant components and no longer had the sensation of pins and needles.

Fifteen hours later the patient still had no return of the pins and needles and no cramping feeling; thus this relief outlasted any objective effects of the local anaesthetic. Scraping a pin across the skin supplied by the median nerve no longer caused the unpleasant dysaesthesiae, but scraping it across the skin below the clavicle caused them.

An incomplete block of the median nerve stopped spontaneous paraesthesiae and the unpleasant dysaesthesiae produced by firm stroking of the skin; the block was differential, in that it affected mainly the sympathetic fibres and those subserving pain. The relief was not restricted to the median territory but extended from the fourth to the seventh cervical dermatomes. The effect outlasted the analgesia induced by the local anaesthetic solution.

J.N. (National Hospital No. 81349). - This 36-year-old man was admitted to the National Hospital in November, 1958. Six months before admission a lobe of the left lung had been removed for carcinoma of the bronchus. A few weeks after the operation, the patient began to have pain down the inner side of the left forearm, extending into the fourth and fifth digits, where he also developed tingling and numbness. Two months beforê iv admission a pain of similar quality and distribution hag $\underline{G}$ appeared in the right upper limb. Weakness develope $\$$. rapidly in the left and then in the right hand. Two weeks before admission the patient had developed severe pait $z$ between the scapulae and weakness of both lower limbs.

On examination, he had much weakness of both uppew limbs, more of the left than the right, and wasting of the $\vec{\varphi}$ muscles of both hands and of the left forearm. The biceps and triceps jerks on the left were absent. There was weakness of both lower limbs, both plantar responses were of the Babinski type, and there was bilateral ankle clonus. All forms of sensibility were diminished over the last three fingers and on the distal part of the ulnar aspect of both forearms. There was some loss of pain and touch sensibility over the trunk and lower limbs below the level of the fourth thoracic segment.

Lumbar puncture revealed a partial block; the cerebrospinal fluid contained 4 cells and $0.6 \mathrm{mg}$. of protein per $\mathrm{ml}$.

In this patient the carcinoma had spread into the spinal canal, compressing the cord. Most of the pain was in the left upper limb distal to the elbow; it was associated with diminution of sensibility in the seventh 3 and eighth cervical dermatomes.

Protocol of Experiment.-Before the injection to block the ulnar nerve, the patient was in obvious distress and $\bigcirc$ his face was pouring with sweat. The pain was distal to the elbow, and was maximal in the ulnar territory of the $\frac{D}{0}$ hand and along the ulnar border of the forearm. He located it as deep, "right through the fingers", but not $N$ in the thumb or index finger. The ulnar nerve was injected in its groove with $2 \%$ lignocaine. Even before $\mathrm{N}$ the patient told us that his pain had been relieved, it was $N$ obvious, from the ceasing of the sweating on the face and 
the other signs of distress. Within five minutes of the injection, there was complete loss of sensibility throughout the ulnar distribution. The pain disappeared completely from this limb and the forearm and hand felt "comfortably warm"; in contrast, the right upper limb felt cold and he became more aware of pain in the right upper limb, which before the injection had been minimal. The pain remained absent from the left upper limb for about two hours.

Blocking the ulnar nerve stopped the pain in the left forearm; this pain had been present in a more extensive area than the cutenous distribution of the ulnar nerve. The pain-relieving effect outlasted the duration of the local anaesthetic for about 30 minutes.

G.N. (National Hospital No. 52599).-This 37-year-old man was in the National Hospital in 1954. He had complained of a varying state of clumsiness of the right limbs, attacks of unsteadiness, and of slurring of his speech. The course of the disorder had been characterized by remissions and exacerbations. He was found to have, besides the obvious slurring of speech, a mild right hemiparesis. The cerebrospinal fluid contained 15 cells and $0.55 \mathrm{mg}$. of protein per $\mathrm{ml}$. and the Lange curve was 122211000; the Wassermann reaction in blood and cerebrospinal fluid was negative.

His unsteadiness and dysarthria gradually improved; but in September, 1957, he developed an intense burning pain in the fourth and fifth digits and along the medial border of the left hand. During the first few months of 1959 his gait became unsteady and he developed blurring of vision. It was then found that visual acuity was reduced and that the right optic disc was swollen; there was nystagmus, an intention tremor in the upper limbs, and both plantar responses were of the Babinski type; Lhermitte's sign was also present. Appreciation of two points, passive movements, and vibration was diminished on the fourth and fifth digits of the left hand; two-point discrimination was equally affected on the median and ulnar cutaneous distribution of the fourth digit. When the fifth, fourth, and medial side of the third left digits were slightly scratched with a pin, the patient experienced an unpleasant spreading sensation like an electric shock; this was not an exaggeration of his constant burning pain.

The diagnosis was considered to be disseminated sclerosis, and the pain was thought to be due to a plaque in the eighth cervical posterior root entry zone.

Protocol of Experiment.-The left ulnar nerve was injected with $2 \%$ lignocaine in the ulnar groove. Within three minutes, all the burning pain left the fifth digit, and within five minutes it had all gone from both digits. At this time there was complete loss of sensibility within the ulnar distribution. As sensibility returned to the ulnar area, the pain returned. By the time sensibility was fully normal, the pain was at its full intensity, and scratching with a pin caused the usual dysaesthesiae.

Twelve days later, the patient returned to say that since this injection the base of the little finger had been free from pain and that there was also less pain in the fourth finger than before the injection.

The ulnar nerve was again injected in the ulnar groove with $2 \%$ lignocaine. As the total anaesthesia and motor paralysis came on, so the pain went away. One hour later sensibility returned except for slight diminution in the appreciation of touch and pin prick; pricking no longer gave the usual dysaesthesiae. The burning pain remained absent.

Two weeks later the patient returned to say that since the previous injection of the ulnar nerve none of the burning pain had returned. He had, however, a deep ache in the fourth and fifth digits and he wondered if this had been present before and that he simply had not noticed it on account of the burning pain. On examination of the digits it was found that scratching with the pin no longer caused the dysaesthesiae, but gave a normal sensation of being scratched, though to a slightly less than normal degree.

Two months later the patient was still free from the burning pain. When the skin of the fourth and fifth digits was stroked with a pin there were no dysaesthesiae: "it just feels rough". The deep pain was present, but the patient said he might go four or five days without having it.

It was considered that the pain and dysaesthesiae in this case were due to a plaque in the root entry zone of the eighth cervical posterior root. The situation of dysaesthesiae and of the pain was typical of a lesion affecting the eighth root and not of the ulnar nerve; yet blocking the ulnar nerve completely stopped the pain throughout the whole area. A second blocking of the nerve gave lasting relief of the burning pain, but apparently unmasked a deep pain. After these injections the sensibility of the affected digits became much more normal; although the appreciation of sensory stimuli remained slightly diminished, the painful dysaesthesiae were no longer present.

A.R. (National Hospital No. 83923). - This 56-year-old woman was admitted to the National Hospital in March, 1958. Eighteen months before admission she had become aware of a sensation of pins and needles in the third, fourth, and fifth digits of the right hand; it had come on insidiously and was present constantly. One year before admission she had had an episode of vertigo, and after this, walking had become unsteady. Seven months before admission the right lower limb had suddenly become weak, and since that time the weakness had gradually become more severe.

When examined in the hospital there was nystagmus of rotatory type on looking to both sides, the tendon reflexes were more active in the right limbs than in the left, and there was a spastic paresis of the right lower limb. In the right upper limb vibration was felt to a diminished degree on the ulnar border of the hand and wrist, and two points were appreciated at a distance of $8 \mathrm{~mm}$. on the right fourth and fifth digits compared with $5 \mathrm{~mm}$. on the left. No form of stimulation of the 
fingers had any effect in intensifying or altering the pins and needles sensation. The cerebrospinal fluid contained 2 cells and $0.45 \mathrm{mg}$. of protein per $\mathrm{ml}$.; the Lange curve was normal and the Wassermann reaction negative. A myelogram was normal.

The diagnosis was considered to be disseminated sclerosis, and the painless pins and needles sensation were thought to be due to a plaque affecting the fibres of the funiculus cuneatus derived from the seventh and eighth cervical dermatomes.

Protocol of Experiment.-The patient was experiencing the usual constant pins and needles in the right third, fourth, and fifth digits before the experiment. The right ulnar nerve was injected in the ulnar groove with $1 \%$ procaine. Within 10 minutes the pins and needles sensation had completely gone. The ulnar nerve block was incomplete. The patient had a mild feeling of tightness in the ulnar distribution. This side of the hand was warm and fully vasodilated; there was a diminution in the sensation of pain on pricking and only very slight, if any, diminution in touch sensibility. One hour later the feeling of tightness was diminishing and the pins and needles sensation was returning, and one and a half hours after the injection the feeling of tightness had gone and the pins and needles sensation had returned to its original intensity.

The paraesthesiae and the type of loss of sensibility were thought to indicate a lesion of the fasciculous cuneatus, involving particularly the fibres from the seventh and eighth cervical segments. Blocking the ulnar nerve stopped the paraesthesiae completely. This effect persisted as long as the local anaesthesia lasted.

H.G.-This 29-year-old man was admitted to the Veterans Administration Hospital, Pittsburgh, in September, 1959. He was complaining of weakness in the right upper and lower limbs and pain in the right hand. He had had pain in the right hand and forearm for 10 years, and over the same period the right upper limb had become progressively weaker and the right lower limb had become clumsy. He had occasionally burned his right hand or fingers while smoking and yet had felt no pain. One year before admission to the Veterans Administration Hospital, a laminectomy had been carried out to lay bare the spinal cord from its first to its fourth cervical segments; no lesion had been found and the cord had appeared quite normal.

In September, 1959, he complained of pain of a boring, aching kind, situated deep inside the palmar and dorsal aspects of the hands; the previous pain in the forearm had gone. This pain was unaffected by movements of the hand, shoulder or neck, by stroking or scratching the hand, or by any manoeuvre designed to increase it. Examination showed that there was almost complete loss of sensibility of pain and temperature on the right, from the second cervical to the sixth thoracic segments inclusive; in this region touch and vibration were normally felt and the sense of position and movement was unimpaired; but two-point discrimination was slightly impaired on the right hand and digits. In the right upper and right lower limbs there was weakness and increased tone. The deep reflexes were absent in the right upper limb; in the right lower limb they were pathologically increased, there was ankle clonus, and the plantar response was of the Babinski type. There were scars due to previous burns on the right hand and fingers.

Lumbar puncture revealed no block; there were 3 lymphocytes and $0.3 \mathrm{mg}$. of protein per $\mathrm{ml}$; the Wassermann reaction was negative.

The diagnosis was considered to be syringomyelia; the deep type of pain was thought to be due to involvement of fibres in the posterior horn of the sixth, seventh, and eighth cervical segments.

Protocol of Experiment.-The right ulnar nerve was injected in the ulnar groove with $2 \%$ lignocaine on three different occasions. The results on each occasion were the same. Within five minutes of the injection, all pain left the entire hand. The pain returned about an hour after the injection, coming back as the motor paralysis went off. The relief of pain never outlasted the duration of the anaesthesia. On each occasion the removal of the pain followed the same course: it first went from the region of the ulnar distribution, and last went from the dorsal part of the lateral half of the hand.

The pain of a deep kind, typical of syringomyelia, located deep to the sixth, seventh, and eighth dermatomes was completely relieved by blocking the ulnar nerve. This effect persisted as long as the local anaesthesia lasted.

G.B. (National Hospital No. 86061).-This 62-year-old woman was admitted to the National Hospital in May, 1959. Her main complaint was of a "scalding pain" in both buttocks and down the right lower limb, which had been present for two years. In January, 1957, the left lower limb had "suddenly given out" and she had fallen; over the next four days she had continuous pain in the back, the abdomen, and down both lower limbs. She was admitted to a local hospital where severe weakness and loss of sense of position in the left lower limb was found, with analgesia over the right side of the body below the fourth thoracic dermatome. Lumbar puncture revealed no block; there were 3 lymphocytes and $0.8 \mathrm{mg}$. of protein per $\mathrm{ml}$.; the Wassermann reaction was negative. When she left her local hospital five weeks later the movements of the left lower limb were improving, much sense of position and movement had been regained, and all pain had gone. In June, 1957, she first developed the scalding pain in both buttocks and down the right lower limb into the heel, which had persisted to the time of admission. She found also that from the waist down, particularly on the right side, any touch caused an unpleasant "queer" feeling; this was not the same as the spontaneous burning pain. On examination, she was found to have a spastic paresis of the left lower limb, associated with diminution in the sense of position and movement, and inability to feel vibration. Below the fourth thoracic dermatome all forms of cutaneous sensibility were abnormal. On the right, pain sensibility was almost absent below the third lumbar dermatome; between the third lumbar dermatome and the fourth 
thoracic dermatomes, pinprick caused a most unpleasant kind of pain-"it seems to go in further and be more pointed"; here the sensibility of warmth and cold were similar, being absent below the third lumbar dermatome, and abnormal below the fourth thoracic dermatome. On the right below the twelfth thoracic dermatome tactile stimuli gave a tingling sensation; this was most marked where the loss of pain sensibility was greatest, and there such stimuli also gave an unpleasant raw sensation. On the left side of the body between the sixth and the twelfth thoracic dermatomes the sensation of touch was reduced and distal to these segments it was very much reduced; in the left buttock, touch produced a sensation of tingling.

It was considered that the lesion giving rise to this clinical picture was an intramedullary lesion of the left side of the cord, extending from the sixth to the twelfth thoracic segments; this was thought to be some sort of vascular accident. As the patient had recovered from the immediate acute effects, the scalding pain had come on gradually. The region in which she had this pain most severely was below the third lumbar segment, in the area where pain and thermal sensibility were most diminished and where tactile stimulation gave the unpleasant raw sensation. It was observed that she had the same pain in the left buttock, where, although tactile stimulation gave a similar raw sensation, there was no change in normal appreciation of painful or thermal stimuli. This finding was difficult to explain satisfactorily.

Protocol of Experiment.-A spinal anaesthetic was given in the form of $1 \frac{1}{2} \mathrm{ml}$. of heavy "nupercaine" which was injected between the fifth lumbar and first sacral spines, the patient lying on her right side, with the caudal parts of her body lower than the cranial. It achieved total analgesia bilaterally over the sacral third, fourth, and fifth dermatomes. Tactile sensibility was difficult to judge on the left as it was always so diminished; on the right it was severely diminished, but not absent, over the buttock only. This injection removed all the pain in both buttocks and the right thigh; it had no effect on the pain in the right leg or right heel. The patient had no subjective sensation of numbness and she therefore had no idea of the presence, extent, or of any change in sensibility due to the spinal anaesthetic.

Although the pain was clearly of central origin, anaesthetizing the nerve roots of an area in which the pain was felt removed the pain. In this case the removal of pain did not outlast the duration of anaesthesia. Also blocking some of the nerves of the region in which pain was felt did not remove the pain from the whole region. Indeed, the fact that the patient did not know which regions had been rendered analgesic and anaesthetic, and that her pain went only from the area supplied by the blocked nerves and not from the area supplied by unaffected nerves, shows that the relief of pain was not due to suggestion or to similar mechanisms.

L.R. (National Hospital No. 74975). - This 37-year-old woman was in the National Hospital in 1958. One year previously, a panhysterectomy had been performed for a squamous-cell carcinoma of the cervix uteri. Two months after this operation she had developed a constant pain in the right hip, which extended down the back of the entire lower limb into the dorsum of the foot. This had become so severe that she was taking pethidine three-hourly. She described the pain as "dreadful" and in character "like red hot needles". More recently she had developed foot drop on the right and the right lower limb had become oedematous. There was a large mass filling the retrovesical space, and in both inguinal regions there were large, very hard glands. There was diminution of sensibility in the fifth lumbar and all the sacral dermatomes on the right. A pinprick of $60 \mathrm{~g}$. weight was felt as a blunt touch, but one of $140 \mathrm{~g}$. was felt as painful; it did not feel like a pin, but like a scratch. Cottonwool was felt everywhere except along the back of the leg below the popliteal space. Temperatures of $12^{\circ} \mathrm{C}$. and $50^{\circ} \mathrm{C}$. were not felt in the fifth lumbar or sacral dermatomes, but radiant heat and ice and ethyl chloride spray were felt as such, except in a smaller area on the back of the leg. All muscles on the right supplied by the sciatic nerve and the pudendal plexus were paralysed; the right ankle jerk and plantar response were absent. The flare of the triple response was absent in the fifth lumbar and all sacral dermatomes on the right.

The carcinomatous tissue was considered to be lining the right wall of the pelvis, involving the lumbo-sacral trunk and all the nerves on the right forming the sacral and pudendal plexi; this involvement was at or peripheral to their ganglia.

Protocol of Experiment.-Pain of the usual severity was present throughout the right lower limb, maximal in the buttock and in the sole of the foot. The sciatic nerve was injected with $1 \%$ lignocaine just below the natal cleft. Within five minutes all the pain went, except that in the buttock. It remained absent for one and a half hours.

Blocking the sciatic nerve removed the severe pain from the territory of this nerve; it had no effect on pain felt in regions supplied by other nerves of the sacral and pudendal plexi.

J.H.P. (National Hospital No. 81235).-This 67-yearold man was in the National Hospital in 1958. Eighteen months before he had developed a painful pricking sensation over the anterior surface of the right thigh, and the leg had become weak. In November, 1957, one year before admission, the pain had become worse and it consisted then of knife-like jabs of pain in the anterior thigh. He was admitted to the Atkinson Morley Hospital. There Mr. L. S. Walsh made a diagnosis of prolapsed intervertebral disc at the second and third lumbar level, and decided to operate. At the operation he found that the dura mater was compressed up against the lamina of the third lumbar vertebra, and the disc between the second and third lumbar vertebrae was abnormally soft and was causing a marked bulge into the spinal canal. A number of fragments of this disc were removed; after their removal there seemed to be a 
satisfactory decompression of the spinal roots. This operation was followed by an uneventful convalescence; the strength of the leg improved and the loss of sensibility regressed, there remaining a very slight loss in the third and fourth lumbar dermatomes. The pain at first was relieved but it began to return about six weeks after this operation.

When the patient came into the National Hospital in September, 1958, the pain was located in the distribution of the cutaneous branches of the femoral nerve. It was worse than it had been before removal of the prolapsed disc and more extensive. There was a continuous tingling in the skin and a deep, burning pain beneath the skin; in addition, whenever this area of skin was touched, the stimulation gave rise to little electric-shock-like sensations.

There was slight wasting and weakness of the right quadriceps and weakness of the flexors of the thigh; raising the fully extended right lower limb caused pain in the lower spine. The right knee and ankle jerks were diminished, the plantar responses were normal. There was loss of sensibility in the distribution of the anterior femoral cutaneous nerve, and slight loss in that of the lateral femoral cutaneous and saphenous nerves. In the former territory pinprick was felt only as blunt touch, in the latter territory, it was slightly diminished; on the dorsum of the foot a very slight loss to pinprick was detected. On the front of the thigh sometimes a very heavy pinprick gave a sensation like a local electric shock. Cottonwool touches were felt to a diminished degree in the fourth and fifth dermatomes. The cerebrospinal fluid contained 4 cells and $1 \mathrm{mg}$. of protein per $\mathrm{ml}$. On myelography changes suggesting arachnoiditis were seen in the region of the former operation.

A sphygmomanometer cuff was placed on the left upper limb in order to render this limb ischaemic for 30 minutes, to induce the various sensations occurring during and after circulatory block; this was done so that the patient could compare these induced sensations with his spontaneous ones, and to enable him to give us a better concept of his pain and paraesthesiae. He reported that the pain in the fingers induced at the end of the period of ischaemia when only $\mathrm{C}$ fibres are conducting was identical with his constant pain in the thigh. He also stated that he sometimes got the sensation of pseudo-cramp in the thigh and the pricking part of the post-ischaemic paraesthesiae.

The diagnosis of prolapsed intervertebral disc had been confirmed at operation. Although much disc tissue had been removed, both the symptoms and signs returned in at least as severe a form as they had been before operation.

Protocol of Experiment.-An injection of $1 \%$ lignocaine with hyaluronidase was given subcutaneously into the region supplied by the medial femoral cutaneous nerve. By the time this area was completely insensitive to tactile and painful stimuli, all the pain had gone from the right lower limb; the only pain remaining was in the back. Thus the pain had gone from the entire limb, not only from the distribution of the cutaneous branches of the femoral nerve; furthermore he found he could flex the knee without getting the unpleasant tight feeling that this movement usually produced. The pain remained absent for three hours, which was longer than the duration of the local anaesthesia.

On another occasion, an attempt was made to inject the right femoral nerve in the femoral triangle with $1 \%$ lignocaine. This injection, however, blocked the muscular branches to the quadriceps and the saphenous nerve; the anterior femoral cutaneous nerve escaped, Nevertheless as soon as the territory of the saphenous nerve was insensitive to touch and pinprick, the pain in the lower limb went. It is to be noted that the area of maximal pain and abnormality of sensibility, that of the anterior femoral cutaneous nerve, was unaffected by the injection, except for slight loss of sensibility in its most distal distribution; and the skin of this region retained its abnormal characteristics with regard to sensibility. And yet this injection removed all the pain in the lower limb. Further, the effects of this injection in relieving pain lasted 48 to 60 hours. Sixteen hours after the injection the following change in sensibility was noted. The injection had been carried out by one of us unknown to the other. The latter, visiting the patient 16 hours after blocking of the saphenous nerve, found an improvement in the sensibility of the skin in the distribution of the anterior femoral cutaneous nerve; although objectively there was no change, the patient when he dido feel a hard pinprick here no longer felt it like a little electric shock but felt it like a normal painful and pointed pin.

In this patient a lesion, proved at operation, was affecting the second, third, fourth, and to some extent the fifth lumbar roots. Blocking of impulses either from the cutaneous distribution of the seconde⿳亠丷厂 and third posterior root or from that of the fourth root removed the pain throughout the distribution of all the roots; further, after one of these injections the pain remained absent for 48 hours; and anaesthetizing the territory of the fourth lumbar dermatome improved the sensibility of the second and third lumbar dermatomes.

\section{Discussion}

Blocking an afferent pathway at a point distal to the site of a lesion stops the spontaneous sensations which result from the lesion. It is reasonable to assume that a lesion situated on an afferent pathway is the originator of the discharge of impulses in the contiguous nerve fibres and that this is the cause of the "spontaneous" sensations experienced; hence it is somewhat surprising to discover that the apparently less probable alternative theory is correct: that the pain and paraesthesiae are due to impulses arriving from the periphery, which are somehow altered by the lesion. Since we have no further evidence to present on the mechanism of the pain in these cases, there seems little point in 
suggesting various conjectures to account for it. It is necessary, however, to discuss whether the phenomena reported here can be accounted for entirely by events at the site of the lesion on the nerves or whether they can be accounted for by invoking factors in the central nervous system.

It is possible that the pathological lesion of the nerves gives rise to an artificial synapse, similar to that produced experimentally by Granit, Leksell, and Skoglund (1944). They showed that in cats, if a nerve is cut and the motor root is stimulated, action currents can be picked up from the sensory root; and also they can be picked up from the motor root when the sensory root is stimulated, though in this case the stimulus needs to be much stronger. The place where the transmission from one lot of nerve fibres to the other takes place, seems to be at the cut end of the nerve. In order to obtain such an artificial synapse they then found that it was unnecessary to cut the nerve across; a ligature round the nerve, tight enough not to damage normal distal conduction, suffices to make such a synapse. It may be suggested that with naturally occurring lesions impulses from the periphery arriving at the site of the lesion become switched into fibres whose central terminations end in parts of the brain where pain and paraesthesiae are experienced; when impulses are blocked from reaching the site of the lesion, impulses cannot be switched to these fibres and so there is silence. A similar mechanism to account for causalgia has previously been suggested by Doupe, Cullen, and Chance (1944) and by Nathan (1947).

But such a mechanism cannot account for all the phenomena reported here. For the following observations cannot be explained without considering that blocking impulses from the periphery cause some changes in the central nervous system. The first of these observations is that blocking the nervous conduction from a part of a territory in which the pain or paraesthesiae are arising is sufficient to stop the pain and paraesthesiae throughout the entire territory; for this to occur, the region from which the nervous pathway is blocked need not be that in which the pain or paraesthesiae are maximal. An instance of this was J.P., in whom blocking the saphenous nerve removed all the pain in the lower limb, although the pain felt in the saphenous territory was minimal in comparison with that felt in the thigh. In many cases the period of relief of pain or paraesthesiae outlasted the duration of the block of the peripheral pathway; in one case the relief was permanent. And there is also the fact that in the cases where the pain or paraesthesiae have been relieved, the actual sensibility of the skin of the part has been rendered more normal. This applies also to regions of skin within the area of pain but outside the territory of the nerve that was blocked. This improvement in sensibility will not be discussed further here, as it will be treated separately in a later paper.

One might suggest that an explanation of the facts described is that the local anaesthetic solution tracked up the peripheral nerves to the site of the lesion in the nerve root or in the spinal cord. This suggestion seems to us unlikely but not one that can be dismissed out of hand. It would entail the supposition that the anaesthetic solution in adequate concentration reaches the spinal roots, root entry zone, or the spinal cord from a nerve injected at the level of the elbow within a few minutes. Yet it has been shown by Merrington and Nathan (1949) that $2 \mathrm{ml}$. of $2 \%$ procaine solution with adrenaline when injected into the ulnar nerve at the wrist spreads within a few minutes for a proximal distance of at least $8 \mathrm{~cm}$. More careful work on the spread of substances when injected intraneurally has been reported in an important paper by Brierley and Field (1949); they studied the spread of radioactive phosphorus from the sciatic nerves of the rabbit. In one rabbit in which the injection into the sciatic nerve was at the level of the great trochanter of the femur (incidentally at the same level as our injection in L.R.) the tracer substance was found nine minutes after injection in the segments of the spinal cord from which the sciatic nerve takes origin. Thus we suggest that the spread of the local anaesthetic up to the roots or spinal cord is not an impossible explanation of our findings, but it is improbable.

If all the facts reported here cannot be explained by events taking place at the site of the lesion in the nerves, if they cannot be explained by spread of the local anaesthetic to the site of the lesion or into the affected spinal segments of the cord, then we have to conclude that blocking impulses at a peripheral level has definite effects on events in the central nervous system, presumably at a spinal level.

If this suggestion is correct, it is concluded that a state of excessive excitability has been engendered in the central nervous system; and that this state is in a region of the central nervous system to which the nerve fibres from the lesion are running. If there is such a state, it is surprising to find how easily it can be made to subside: half an hour's anaesthesia may suffice to do so for periods of at least two months.

The next question that comes to mind is whether blocking of impulses from the periphery will relieve the pain in all cases where there is a lesion on a nerve, posterior nerve root, or afferent path in the cord. Obviously such a question cannot be answered. The cases reported here are an un- 
selected sequence of cases in which a lesion on an afferent pathway was associated with pain and paraesthesiae. In no cases have we blocked the periphery without obtaining this result. The fact that we have not found any counter-examples does not mean that we believe that they do not occur. Our purpose was to investigate the cases we found where it was evident that the pain or paraesthesiae were arising central to the incoming peripheral nerves; in these few cases the pain or paraesthesiae were stopped by blocking the peripheral pathway.

Over the past 10 years one of us (P.W.N.) has anaesthetized the affected area of skin in many cases of post-herpetic neuralgia; this may be considered to be a similar situation. But here the results are less definite. Usually some of the pain is relieved by subcutaneous injection of local anaesthetic. Certainly if the skin is rendered anaesthetic and analgesic, any pain usually felt in the skin ceases; typically the patient states that the burning hot part of his pain has gone, but the gripping aching and deeper part of his pain remains. This part of the pain is not usually relieved by anaesthetizing the muscles. It is also relevant that in such cases the operation of posterior rhizotomy is notoriously unsuccessful; and this cutting of the roots might be thought of as being comparable to anaesthetizing the peripheral pathway, as was done in these experiments.

\section{Summary}

The pain and paraesthesiae associated with lesions in peripheral nerves, nerve roots, root entry zone, posterior columns, and spino-thalamic tract can be prevented by blocking the nerves distal to the lesion. The relief of pain and paraesthesiae may outlast the duration of the anaesthesiae. Blocking of impulses from a large part of the region supplied by the affected nerves may remove the spontaneous pain and paraesthesiae from the whole of the region.

We wish to thank the physicians and surgeons of the National Hospital, Queen Square, who were kind enough to put their patients at our disposal for this investigation and Dr. E. Arnold Carmichael for his encouragement and facilities to do this work.

\section{REFERENCES}

Brierley, J. B. and Field, E. J. (1949). J. Neurol. Neurosurg. Psychiat., $12,86$.

Doupe, J., Cullen, C. H., and Chance, G. O. (1944). Ibid., 7, 33.

Granit, R., Leksell, L., and Skoglund, C. R. (1944). Brain, 67, 125. Merrington, W. R., and Nathan, P. W. (1949). J. Neurol. Neurosurg. Psychiat., 12, 1.

Nathan, P. W. (1947). Brain, 70145. 